ings and partly to provide funds sufficient to maintain and work them.

There are clearly only two sources from which the very large sums required by the universities can be obtained, and those are (1) Treasury grants and (2) private benefactions.

You have pointed out that the proposed Treasury grant of $1,500,000 l$. included in the Estimates for $192 \mathrm{I}-22$ is quite inadequate, and it is obvious that this must be the case. It is, therefore, to be hoped that careful inquiry into the needs of the universities by the Treasury will result in this sum being substantially augmented. With regard to private benefaction, I think we may look forward with confidence to very liberal response in the near future from generous individuals, and more particularly from wealthy firms interested in the progress of science and education. The action of Messrs. Brunner, Mond, and Co. in setting aside $100,000 l$. as a contribution to the universities is an example which will certainly be followed by other firms who owe much of their success to the work of chemists, engineers, and others sent to them from the universities.

If it were to become a recognised practice for firms who can afford to do so to set aside yearly some comparatively small sum as a contribution to the universities, the combined effort would go far to solve the difficulties in which we find ourselves at the present time.

The University Museum, Oxford, August 22.

\section{Use of Sumner Lines in Navigation.}

May I venture to point out, in the interests of navigational science, that although the article by Capt. Tizard in Nature of July I under the above title is an admirably clear and concise account of the application of Sumner lines in navigation at the date given in his examples, it is scarcely descriptive of the best practice of to-day?

Of the two methods of drawing the lines described by Capt. Tizard, the first, or "original Sumner method," is now merely of academic interest, and is seldom practised outside schools and examinationrooms. Its defects are, first, that each sight has to be worked out twice (once for each of the two assumed latitudes), and, secondly, that it is inapplicable to sights taken near the meridian. It may also be remarked that unless the two assumed latitudes are very close together, the true circle of position may differ considerably from the straight line joining the two points found on the Mercator chart.

The second method described by Capt. Tizard, usually known as the "chronometer method," is still used to some extent. It avoids the double working out of each sight, but gives good results only for observations taken on large bearings; it is inapplicable to sights taken near the meridian.

For observations near the meridian what is cailed the "ex-meridian method" may be used to draw the position-lines. In this method the longitude is assumed, and the sight is worked out as a latitude observation; the position-line is then drawn, at right angles to the bearing, through the point where the meridian of the assumed longitude is cut by the parallel of the observed latitude. This method gives good results for sights near the meridian, but fails on large bearings.

A combination of the last two methods is sometimes employed, the sights near the meridian being worked out by the "ex-meridian method," and those on large bearings by the "chronometer method." This combined procedure has been advocated by several writers, especially by Capt. Blackburne, who NO. 2652 , VOL. IO5] undertook the immense labour of computing tables specially adapted for the purpose. The main objec. tion to it is that the procedure is not uniform for all sights.

A much better method of drawing the Sumner lines than any of the above, and one which seems destined to replace all others, being now in extensive use by navigators of all nations and recognised as the standard method in the Royal Navy, is known as the Marcq Saint-Hilaire method, or the "new navigation." It consists in assuming a dead-reckoning position in both latitude and longitude, and then finding how much the observed zenith-distance of a heavenly body differs from that calculated on the assumption that the dead-reckoning position was correct. The difference between the observed and calculated zenith-distances is laid off from the assumed position in a direction to or from the observed object (according as the observed zenith-distance is less or greater than the calculated one), and the position-line is then drawn through the point so found at right angles to the bearing. The great advantage of this method is that it is perfectly general; it gives equally good results whatever the bearing of the object sighted.

Though called the "new navigation," the Marcq Saint-Hilaire method of drawing the Sumner lines is by no means a recent invention, having been used in the French Navy for more than forty years. Its advantages were advocated so long ago as 1888 by that indefatigable worker for the advancement of navigation, the Rev. William Hall, R.N., and have since been frequently pointed out by other English writers on navigation. Its superiority over all other methods for drawing the Sumner lines or position-lines being indubitable, there is a iittle difficulty in understanding its comparative neglect by British navigators up to recent times. One reason, no doubt, is conservatism; the British seaman usually prefers to use time-honoured methods with which he is familiar rather than to adopt new-fangled notions, and fears to risk his ship by the possibility of making a mistake in a process with which he has not been made acquainted during his early training. Another reason which operated powerfully until within the last twenty years or so was the absence of any tables for facilitating the calculation of altitudes comparable in scope with the tables of Davis and Burdwood, which so greatly helped in the rapid reduction of sights by the "chronometer method." This last difficulty was removed by the publication of the excellent "Altitude Tables " of my namesake, the Rev. F. Ball, M.A., of the Roval Navy, and at the present time it is just as simple a matter to work out sights by the "new navigation," with the aid of these tables, as it was to work the old "chronometer method" with the help of Burdwood and Davis.

Until within the last decade it was seldom worth while to attempt to fix a ship's position at sea within a mile or two, because so long as the longitude, whether found by Sumner lines or by any other method, was dependent entirely on the Greenwich time as found by the transport of chronometers over long distances, it was usually impossible to be sure of the longitude within that amount, no matter how accurately star observations were made. This difficultv affected the hydrographic surveyor as well as the navigator; and, indeed, it provides the explanation why so many charted longitudes-down the Red Sea, for instance-are in error by a mile or more. But nowadays, when wireless time-signals enable the error of a ship's chronometer to be found daily with an accuracv of a few tenths of a second anywhere on the seas, there is no reason why the longitude should 
ever be uncertain by so much as the tenth of a mile, provided only that sights can be taken with a corresponding degree of accuracy. Thus the advent of wireless telegraphy, by removing at one stroke the most serious of all pre-existing limitations to precision in the results, has made it worth while to improve the methods of position-finding at sea. Simultaneously progress has been made in the construction of charts and instruments adapted for navigation, giving to the navigator another stimulus towards attaining that refinement of method by which alone he may hope to steer his ship from port to port not only in safety, but also with that economy of time and fuel which is demanded by modern competition.

As regards the number of position-lines required to determine a ship's position, it is obvious that if only two sights are taken, no matter how favourable the angle at which the position-lines cut each other, the position found will be correct only if the observations are free from instrumental and other errors, and if dip and refraction are correctly allowed for. With only two sights a large unknown centring error in the sextant employed, or abnormal refraction, or a mistake in one of the sights, may render the position found quite false, and there is no: means of detecting the error. If the two sights are not simultaneous or nearly so, there will, of course, be an added uncertainty in the position due to the difficulty in accurately estimating the ship's run in the interval. If three sights are taken constant errors can be eliminated, but accidental errors cannot be readily detected. If four sights are taken, however, as nearly as possible simultaneously, on bearings differing by approximately $90^{\circ}$, not only will a constant error of even two or three minutes in the measured altitudes, or in the allowance for dip, be without influence on the accuracy of the result, but if a mistake has been made in one of the sights the fact can readily be detected. This is a powerful argument for making the astronomical determination of a ship's position depend, whenever possible, on at least four Sumner lines or position-lines deduced from observations of four stars differing by approximately $90^{\circ}$ in bearing.

It can easily be proved geometrically that when the altitudes of three or more stars have been equally accurately observed the most probable position is the centre of that circle which most nearly touches all the position-lines, and in which the directions of the stars from the points of contact are either all towards or all away from the centre; also, that the radius of the circle gives the amount of any constant error in the observed altitudes, whether due to errors of the sextant employed or to error in the assumed dip of the horizon or refraction. If with more than three sights no circle can be drawn satisfying the condition of approximately touching all the position-lines, while at the same time having the star-directions from the points of contact pointing either all towards or all away from its centre, then it is certain that a mistake has occurred in one or more of the observations; either an altitude or a time has been wronglv recorded or one of the stars wrongly identified, or else there has been a mistake in the calculation for one or more of the sights.

The importance of considering the directions of the stars, as well as the position-lines themselves, is well illustrated by reference to the first of the examples given by Capt. Tizard. If the non-intersection of the three position-lines in his Fig. I is due to a constant error in all the altitudes, caused either by instrumental error or by error in the tabular allowance for dip or refraction (as will usually be the case in sights taken by a practised observer), then NO. 2652 , VOL. IO5] the true position is not, as might at first be thought, within the little triangle formed by the crossing of the lines, but outside it; and the true longitude is not $145^{\circ} 4^{\prime}$, as Capt. 'Tizard concludes, but $145^{\circ} 5^{\prime}$. For, as will be seen by Fig. I, on which I have indicated the star-directions by arrows, no other circle than the one shown can be described so as to touch all three position-lines, while the three star-directions from the points of contact point either all towards or all away from its centre.

So great is the importance of accuracy in the fixing of the ship's position at sea in modern navigation, and so well is the "new navigation" with four position-lines crossing at about $90^{\circ}$ adapted to secure this accuracy, that at the recent International Hydrographic Conference in London it was proposed by an eminent authority, Comdr. Alessio, of the Royal Italian Navy, that it would be desirable for the conference to prescribe as a fundamental rule of navigation that "the normal astronomical determination of a ship at sea must be made with the method of four Sumner lines by observing four stars the positionlines of which cut at approximately $90^{\circ}$." It was decided that the prescribing of rules for navigation did not fall within the scope of the IgIg Conference,

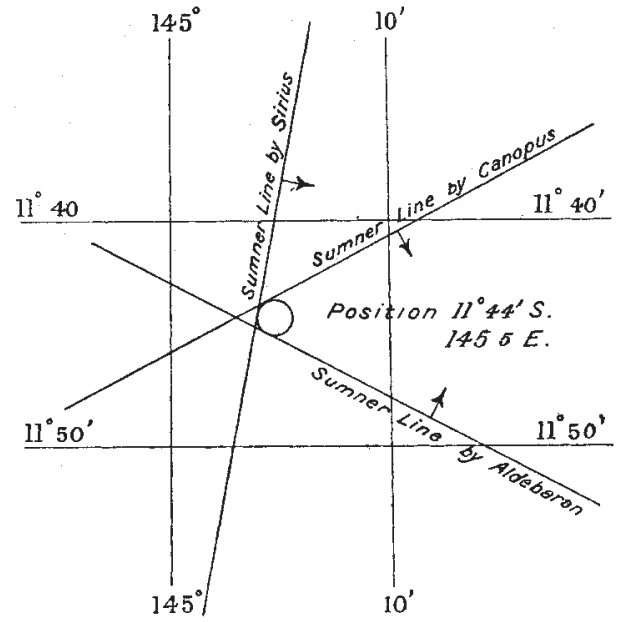

Fig. I.

and consequently the matter was not further discussed. But there can be no doubt that if navigators of all nations could be persuaded to follow so excellent a rule as that suggested by Comdr. Alessio, it would add greatly to the safety of shipping. The method is so simple, and affords such security against error, that if it were once systematically taught in schools of navigation and included in the Board of 'Trade requirements for masters' certificates, it would probably by its own merits displace all other processes for fixing positions at sea under normal conditions. It would, of course, still be advisable to retain the ordinary meridian or ex-meridian sights for latitude and the morning or afternoon sights for longitude as a stand-by against the possibility of clouds or fog interfering with the twilight observations of stars, but whenever the suggested rule could possibly be followed it could be trusted to give far more accurate results than any observations of the sun.

A word may perhaps be added as to the manner of calculating the altitudes in the "new navigation." Comdr. Alessio (Report of the International Hydrographic Conference, London, 1919, p. 230) recommends logarithmic calculation with five-place tables, 
using a formula which permits of a ready check. This only takes about five minutes for each sight, and is, no doubt, the best way; in fact, it is the only safe way where a very considerable degree of precision is aimed at. But most navigators prefer to avoid computation so far as possible by the use of tables, and in ordinary circumstances the altitude tables used in the Royal Navy will give sufficiently accurate results. The great defect of the tabular method is that one has to round off the deadreckoning latitude to the nearest degree for the assumed position in order to enter the tables, and consequently the position-lines may extend over so great a distance on the chart that their curvature cannot properly be neglected. With logarithmic calculation, on the other hand, the actual deadreckoning position can be taken as the assumed position, and the position-lines will then be so short that their curvature can be neglected without any perceptible loss of accuracy.

It may not be out of place to remark in conclusion that the utility of the Sumner line or position-line principle is not confined to position-fixing with a sextant at sea. I have shown in two necently published papers ("Notes on the Working of the New Navigation," Cairo, Igr8, and "The Prismatic Astrolabe," Geographical Journal, July, I919, p. 37) that the "new navigation" is capable of useful application on land in conjunction with theodolite observations and wireless time-signals, and that determinations of geographical position of very considerable accuracy may be made in this way. The method has since been put into practice by Dr. Hamilton Rice on exploratory land surveys in South America (see the Geographical Journal for July, p. 59) with satisfactory results.

Survey of Egypt, Cairo, July 24.

\section{Relativity and Hyperbolic Space.}

OBSERVATION tells us that while gravitation dominates the history of a lump of matter moving in the vast ocean of free æther, it has practically no effect on the history of a pulse of light in similar circumstances. Since last mail I have investigated the bearings of space being hyperbolic on light-rays.

The central-projection map of the space, used before, in which $r=\tanh \theta$, where $r$ is the radius vector of the map and $R \Theta$ the radius vector in the space, will be called a gnomonic map; planes are mapped as planes. If the projection used be given by $r=2 \tanh \frac{1}{2} \theta$, the map will be called stereographic; small regions are mapped in correct shape, spheres and planes as spheres, and the two sheets of a pseudo sphere as two spheres intersecting and making equal angles with the sphere representing the median plane, in a circle lying on the absolute, $r=2$. (A pseudo-sphere is the locus of a point at a given distance from a given plane, called its median plane. The characteristic of the mapsphere which represents a plane is that it cuts the absolute $r=2$ orthogonally.) A point $(x, y, z)$ on the gnomonic map becomes $\left[x /\left(1+\frac{1}{4} r^{2}\right), y /\left(x+\frac{1}{4} r^{2}\right)\right.$, $\left.z /\left(I+\frac{1}{4} \gamma^{2}\right)\right]$ on the stereographic map.

The behaviour of a ray of light is fully described by saying that its path on the gnomonic map may be put in the form $x^{2} / a^{2}+y^{2}=\mathrm{I}$, where $a$ is less than $I$, and that the eccentric angle is $t / R$, where $t$ is co-ordinate time. This ellipse really represents the two branches of a pseudo-circle; the ray goes out to infinity (in the space) along one branch and returns along the other, the complete circuit having the period $2 \pi R$. The median line of the pseudo-circle NO. 2652 , VOL. IO5] passes through the origin-that is, through the observer.

If from a given point rays start in all directions there will be a definite wave-front. For a finite time before $t$ attains the value of a quarter-period, $\frac{1}{2} \pi R$, this front will form the single sheet of a true sphere the centre of which recedes to infinity, whereupon the front develops the two sheets of a pseudo-sphere, the one proceeding in the same direction as before, and the other, together with the median plane, returning from infinity, having been reflected back by the absolute. By the time $t=\frac{1}{2} \pi \mathrm{R}$ the median plane has just reached the origin, and the reflected sheet is chasing both the other sheet and the median plane back on the way to infinity. In the next quarterperiod these motions are reversed in order of time, in direction of motion, and in position relative to the origin. At the time $t=\pi \mathrm{R}$ the front has contracted down to a point focus situated on the opposite side of the origin from the radiant point at a distance equal to that of the point. At the time $t=2 \pi \mathrm{R}$ the original circumstances recur, and everything is about to be repeated. A ray always moves normal to the front, although the centre of the true sphere and the median plane of the pseudo-sphere themselves move from and to infinity in a finite time.

All these motions can be exactly imitated in Euclidean space. Let, at a given point in such a space, the velocity of light be $I+r^{2} / 4 R^{2}$, the same in all directions, and let the sphere $r=2 \mathrm{R}$ be a perfect reflector. Then light will in this medium behave exactly as does the light in the stereographic map (when the scale of that map is increased in the ratio of $\mathrm{R}$ to $\mathrm{I}$ ). Indeed, this seems the easiest method to get the differential equation of the path of a point in the hyperbolic space, for which $\int d t$ is stationary. I may remark, however, that when the equation is obtained, later work is much simplified by changing the dependent to a form corresponding to the gnomonic map.

In the stereographic map the rays after an even number of reflections, by the absolute form a system of coaxial circles through the radiant point and that point on the opposite side of the origin which is inverse to the sphere $r=2$. (For radiant point let $0=x-a=y=z$. Then for the second point mentioned it is meant that $0=x+4 / a=y=z$. Ordinary inverse point would be $0=x-4 / a=y=z$.) After an odd number of reflections they are similarly related to the focus mentioned above. The fronts are the spheres cutting these coaxial circles orthogonally.

University of Tasmania, June Io. Alex. McAulay.

\section{The Antarctic Anticyclone.}

In Nature for August 5 Mr. R. F. T. Granger remarks: "The same conditions, i.e. the surface outflow and the central descent of air, exist in Prof. Hobbs's polar ice-cap anticyclone; the only difference is the physical origin.'

In the case of the ice-cap there are other differences as well; the temperature is lower in the case of an ice-cap than in an anticyclone. The ice-cap conditions which resemble those of an anticyclone are, as $\mathrm{Mr}$. Granger says, "surface outflow and the central descent of air." The differences are low temperature, low pressure, and different physical origin. My suggestion was that these differences made it inadvisable to call them both anticyclones.

Tintagel, Kew Gardens Road, Surrey, R. M. DeElay, 